\title{
Current Status of Pediatric Oncology in Iran
}

\section{Mardawig Alebouyeh}

Department of Pediatrics, Shahid Beheshti University of Medical Sciences, Tehran, Iran.

\begin{abstract}
The pediatric hematology and oncology (PHO) services in Iran have steadily improved during the past 10 years. Today, we have specialized PHO services in almost all major cities throughout the country, where 43 board-certified or eligible pediatric hematologist-oncologists are currently giving care to children suffering from cancer or hematological disorders. In this paper, the state of Pediatric hematology-oncology in Iran will be presented.
\end{abstract}

Keywords: Pediatric- hematology-oncology- Mahak- Iran

Asian Pac J Cancer Care, 2 (4), 71-73

\section{Pediatric hematology-oncology in Iran}

The pediatric hematology and oncology (PHO) services in Iran have steadily improved during the past 10 years [1]. Today, we have specialized PHO services in almost all major cities throughout the country, where 43 board-certified or eligible pediatric hematologist-oncologists are currently giving care to children suffering from cancer or hematological disorders. [2] There is an approved fellowship program for PHO at three university children's hospitals (Ali Asghar and Mofid Children's Hospitals in Tehran, and Golestan Children's Hospital in Ahwaz). Only board-certified pediatricians are eligible for this fellowship program and have to pass written and oral examinations for admission. After completing the three-year fellowship training program and passing the final board examination, the qualified specialists have to serve for five years in designated medical centers in different parts of the country according to the current rules, exerted by the Ministry of Health and Medical Education. The present conditions for enrollment in the fellowship program have ensured fair distribution of pediatric hematology and oncology services throughout the country. As both fields of pediatric hematology and oncology are expanding rapidly, the necessity to revise the current fellowship program is already felt, and a separate training program for each specialty should be started in the near future. Concurrent to the improvements in the field of $\mathrm{PHO}$, there has been a good training program for pediatric surgery. Today, seventy-four board-certified pediatric
Submission Date: 05/17/2017 Acceptance Date: 08/28/2017

surgeons are providing surgical care to pediatric patients.

There are a good number of pediatric and orthopedic surgeons with special training and interest in the surgical management of pediatric tumors, who work in tandem with PHO departments and pediatric oncologists. Hematology-oncology postgraduates are encouraged to take advantage of national and international scholarships and postgraduate programs to update and expand their knowledge and experience at specialized centers abroad. There has been a need for special training programs for teaching nursing staff the principles of caring the children with malignancies. Preliminary actions to set up a special training program for interested nurses are currently in process.

\section{Importance and incidence of childhood malignancies}

In affluent countries, childhood malignancies are accounted among the leading causes of mortality in children only after traffic and poisoning accidents. In fact, $0.2 \%$ of all under-15-year-old children (in other words 1 out of 500 children) suffer from cancer [2]. Due to improving socioeconomic conditions and public health services in the past decade in Iran, there is strong evidence for a change of pattern in the incidence of various diseases in children. Current diagnostic and treatment approaches are very promising and approximately $70 \%$ of children with cancer achieve a 5-year survival [2-4]. Iran has a population of over 66 million, of whom $34 \%$ are younger than 15 years. The key point for health authorities and

\footnotetext{
Corresponding Author:

Dr. Mardawig Alebouyeh

Department of Pediatrics, Shahid Beheshti University of Medical Sciences, Tehran, Iran.

Email: alebouyehm@yahoo.com
} 
the public, in general, is that cancer in children is curable and, therefore, it is worthwhile to survey the current status of pediatric oncology services. The majority of children with cancer are referred either to pediatric oncologists or pediatric oncology departments. However, the time lapse between disease manifestation and referral to specialists still remains a matter of concern. This especially holds true for patients requiring diagnostic or therapeutic surgical interventions [5].

\section{Treatment and outcome of childhood malignancies}

Childhood malignancies are treated according to the conventional multimodality protocols which are mostly adopted from accredited medical institutions in the USA or Europe. There is no shortage of anticancer drugs, antibiotics, hematopoietic growth factors, blood products, and even monoclonal antibodies. However, specialized laboratories capable of performing more sophisticated diagnostic procedures e.g. immune-histochemistry, cytogenetic, DNA, viral and molecular studies, and monitoring are limited in number and offer their services mainly in Tehran.

Radiotherapy facilities are conventional and available only in few major cities. Currently, five linear accelerators are in operation in the country: three in Tehran, one in Isfahan, and one in Hamadan. They all have long waiting lists which limit the expected benefits in some malignancies such as Wilms' tumor.

Currently, two bone marrow transplantation centers (BMT-C) are operating in Iran. The "Hematology-Oncology and BMT Research Center" is the major one, and is affiliated with Tehran University of Medical Sciences. It is well staffed and equipped and has established the facilities and techniques to utilize peripheral stem cells and fetal cord blood for respective candidates. The other active BMT-C is affiliated with Shiraz University of Medical Sciences. However, considering the number of referrals, both centers have limited in-patient capacities and, therefore, long waiting lists for BMT candidates. They are also less experienced in dealing with childhood malignancies and giving proper medical and nursing care to pediatric patients. Therefore, efforts should be made to fill this gap and establish BMT-C targeting childhood malignancies as its main focus of activities.

Iranian blood banking and transfusion services, benefiting from an efficient network throughout the country, are competent enough to cover daily blood requirements of the country with reliable products and quality control. They also provide a variety of blood derivatives and laboratory facilities to perform special serology, immunology, and coagulation tests.

There are encouraging reports from single institutions about treatment and follow-up results of childhood malignancies e.g. acute lymphoblastic leukemia, Hodgkin's disease, Wilms' tumor, and retinoblastoma. [6-9] But, in the absence of an effective national cancer registry and cooperative pediatric study groups, comparative demographic, epidemiologic, treatment, and follow-up data are not available. However, according to the childhood cancer statistics, 2,682 children contracted cancer in Iran in 2000 and the death toll from cancer in pediatric age group was 1,703. [10] Considering these alarming data, it will be a challenging and rewarding task for the Iranian Society of Pediatric Hematology and Oncology (ISPHO) to set about tackling such important problems. In fact, ISPHO should help medical and health authorities and support groups to focus on shortcomings and manage a better allocation of resources. It is also within the responsibilities of the ISPHO to establish a central ethics committee and organize study groups to set guidelines for standard diagnostic procedures, cooperative diagnostic and treatment approaches, as well as new modalities. It should further urge the pediatric oncology departments to establish, as the first step, their own hospital-based cancer registries and ethics committees.

Treatment expenses for children suffering from cancer are to a considerable extent covered by insurance services which act under the auspices of the Iranian government. Expenses for BMT are covered by special government funds as well. Local charity groups and organizations, especially in major cities, are keen to provide financial support and psychological assistance to families in need.

\section{"MAHAK", the charity society to support children suffering from cancer}

Tehran, having a population of over 12 million, takes advantage of seven PHO departments. To provide optimal care and treatment to children afflicted with cancer and to help their families to overcome the financial and psychological burdens, we organized in Tehran a voluntary non-governmental (NGO) charity society by the name of "MAHAK" in 1991 [11]. Since then, our volunteers have been fully engaged and integrated into the network of the social services of the respective PHO departments in Tehran. MAHAK's further substantial accomplishments have been: 1) supporting over 7000 children afflicted with cancer; 2) public education about childhood malignancies and their curability; 3 ) evoking social motivation and soliciting public resources; 4) establishment and management of a hostel to accommodate commuting patients and their parents; 5) building a recreation and rehabilitation center with adjacent diagnostic and therapeutic facilities including a linear accelerator; and 6) establishment of a preliminary cancer database and

compiling over 7000 files, thus filling the existing information gap. MAHAK has attained, since the year 2000, a consultative status from the United Nations Economic and Social Council (ECOSOC). We have encouraged and assisted our colleagues in other major cities to organize similar voluntary charity groups.

Based on the clinical experiences gained in the past decade, we shall coherently endeavor to build a better future for the children suffering from cancer.

\section{References}

1. Alebouyeh M. Pediatric hematology and oncology in Iran: past and present state. Pediatric hematology and oncology. 1993;10(4):299-301. 
2. Bleyer WA. What can be learned about childhood cancer from "Cancer statistics review 1973-1988". Cancer. 1993;71(10 Suppl):3229-36.

3. Stiller CA, Allen MB, Eatock EM. Childhood cancer in Britain: the National Registry of Childhood Tumours and incidence rates 1978-1987. European journal of cancer (Oxford, England: 1990). 1995;31a(12):2028-34.

4. Kaletsch U KP, Michaelis J. Jahresbericht 1996 des Deutschen Kinderkrebsregisters Mainz. Mainz: Institut für Medizinische Statistik und Dokumentation; 1996.

5. Khalegnejad-Tabari AAM. Assessment of time lapse between diagnosis and treatment of abdominal and pelvic tumors in children: results from a single institution. INTCR Annual Meeting. Brussels, Belgium. Brussels, Belgium. 2003. Abstract Book. p.

6. Alebouyeh M VP, Moussavi F, et al. Childhood ALL in Iran: results of 20 years treatment and follow-up at a single institution. SIOP Annual Meeting; Amsterdam, The Netherlands.2000. Abstract Book. p.

7. Alebouyeh M, Vossough P. Hodgkin disease in Iranian children. European journal of pediatrics. 1993;152(1):21-3.

8. Vossough P MN, Alebouyeh M. Wilms' tumor in Iranian Children: 20 years treatment and follow-up. SIOP Annual Meeting; Vienna, Austria. Abstract Book. 1996.

9. Vossough P SH, Azarkeyvan A, et al. Retinoblastoma in Iran: results of 10 years treatment and follow-up. INCTR Annual Meeting; Brussels, Belgium. Abstract Book. 2003.

10. Ferlay I BF, Pisani P, et al. Cancer Incidence, Mortality, and Prevalence Worldwide. Version 1.O. IARC Cancer Base No 5 Lyon: IARC Press; 2001.

11. Alebouyeh M VP. Charity organization to support children suffering from cancer (MAHAK). INCTR Annual Meeting; Brussels, Belgium. Abstract Book. 2002.

\section{$\Theta(\odot)$}

This work is licensed under a Creative Commons AttributionNon Commercial 4.0 International License. 